\title{
The Treatment of Avulsion Fracture of the Calcaneal Tuberosity: A New Technique of I80-Degree Annular Internal Fixation
}

This article was published in the following Dove Press journal:

Clinical Interventions in Aging

\author{
Ding $\mathrm{Xu}^{*}$ \\ Weigang Lou* \\ Ming Li \\ Jianming Chen
}

Department of Orthopedic Trauma Surgery, Ningbo No.6 Hospital, Ningbo, People's Republic of China

*These authors contributed equally to this work
Correspondence: Ding Xu

Email xuding83II29@|26.com

\begin{abstract}
Due to the traction of the Achilles tendon and osteoporosis, a large number of reports have shown that a series of complications such as skin flap necrosis and failure of internal fixation after surgery often cause nonunion or malunion of calcaneal tuberosity fractures. At the same time, there is no uniform standard for the operative procedure in the treatment of the avulsion fractures of the calcaneal tuberosity. We presented a new technique for the treatment of avulsion fractures of the calcaneal tuberosity, which is fixed with a 180degree microplate. We aim to provide a simple, safe, and strong internal fixation technique for avulsion fractures of the calcaneal tuberosity as one of the treatment options.
\end{abstract}

Keywords: 180-degree annular internal fixation, avulsion fractures of the calcaneal tuberosity, new technique

\section{Introduction}

Avulsion fractures of the calcaneal tuberosity are rare in clinical work, and mostly occur in elderly patients with osteoporosis and diabetes. There are also some young patients, who are often athletes that are affected by this type of fracture. It accounts for $1 \%$ to $3 \%$ of all calcaneal fractures. ${ }^{1}$ However, the incidence of the avulsion fracture of the calcaneal tuberosity is also gradually increasing due to the increasing age of the population and the increase in the number of patients with diabetes. Fractures of the calcaneal tuberosity are usually avulsion fractures caused by coaxial contraction of the gastrocnemius-soleus complex. The displacement of the fracture block causes local excessive tension or soft tissue irritation, which can easily lead to local flap necrosis. In severe cases, exposure of the calcaneus and Achilles tendon may occur. Because most avulsion fractures of the calcaneal tuberosity are often displaced very obviously, and even spread to the subtalar articular surface, surgical treatment is required. ${ }^{2-4}$ Early surgical treatment can help to avoid the continuous pressure of the fracture on the skin and reduce the incidence of skin necrosis and it can restore the function of the calf triceps early, which is of great significance to the rehabilitation of the patient's ankle function. ${ }^{5}$ Regarding surgical treatment options using internal fixation, including lag screws, Kirschner wires, steel cables, anchors, and steel plates, all of these techniques have their shortcomings as well as strengths. ${ }^{6-9}$ The calcaneal tuberosity is the stop point of the Achilles tendon. Due to the traction of the Achilles tendon and osteoporosis, a large number of reports have shown that 
a series of complications such as skin flap necrosis and failure of internal fixation after surgery often cause nonunion or malunion of calcaneal tuberosity fractures. ${ }^{10,11}$ At the same time, there is a lack of large sample case studies, so there is no uniform standard for the operative procedure in the treatment of the avulsion fractures of the calcaneal tuberosity.

We presented a new technique for the treatment of avulsion fractures of the calcaneal tuberosity, which is fixed with a 180-degree microplate. We aim to provide a simple, safe, and strong internal fixation technique for avulsion fractures of the calcaneal tuberosity as one of the treatment options.

\section{Patients and Methods}

The study was approved by the Ethics Committee of Ningbo No.6 Hospital. Written informed consent was obtained from the participant for the case details and images to be published.

A 61-year-old male patient who was previously healthy suffered a fracture of the left calcaneal tuberosity due to a fall and was treated with open reduction and internal fixation at the local hospital (Figure 1A-C). The operation process went smoothly, and the targeted treatment was given to reduce swelling and prevent infection after the operation. One month later, the patient suddenly felt severe pain in the left heel during ankle joint function training and was unable to move. He came to our hospital for treatment. Physical examination showed obvious swelling and tenderness of the left heel. At the same time, the skin tension was high and the movement of the ankle joint was obviously restricted. The pulsation of the left dorsal artery was reachable without any significant weakening.
The blood supply and sensation of the extremities were normal. The X-ray showed a fracture fragment of the left calcaneal tubercle shifted again and the internal fixation failed (Figure 2).

After perfecting the examination (routine blood test, coagulation function, electrocardiogram and lung CT) and eliminating the contraindications for surgery, we performed surgery on the patient. Following confirmation of consent, the patient was placed in the prone position and a $5 \mathrm{~cm}$ U-shaped incision was made along the posterior ankle dermatoglyph at the level of the calcaneal tubercle after general anesthesia (Figure $3 \mathrm{~A}$ and $\mathrm{B})$. The soft tissue was incised layer by layer to expose the calcaneal tuberosity fracture. First, we reduced the fracture of the calcaneal tuberosity under direct vision and temporarily fixed it with multiple Kirschner wires. Second, we made a soft tissue channel that clinged to the front of the Achilles tendon to place a pre-curved U-shaped micro locking plate (Weigao, Shandong, China) for fixing the calcaneal tuberosity to the calcaneus. The screws on both sides were fixed at 180 degrees. If the avulsion fracture was relatively large and comminuted, a micro locking plate can be added to fix the calcaneal tuberosity. A rigid fixation giving good stability to the fracture was achieved. The wound was closed in the usual fashion. After the operation, we gave infrared thermal physiotherapy to promote the recovery of blood supply around the incision. At the same time, the patient's ankle joint flexion and extension exercises were strengthened under non-weight bearing conditions. We changed the dressing of the patient's incision at an interval of every 3 days.
A

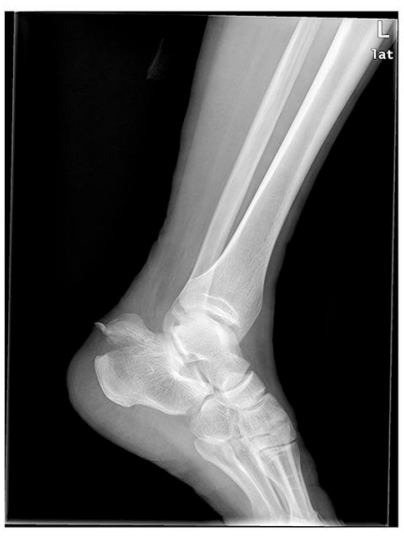

B

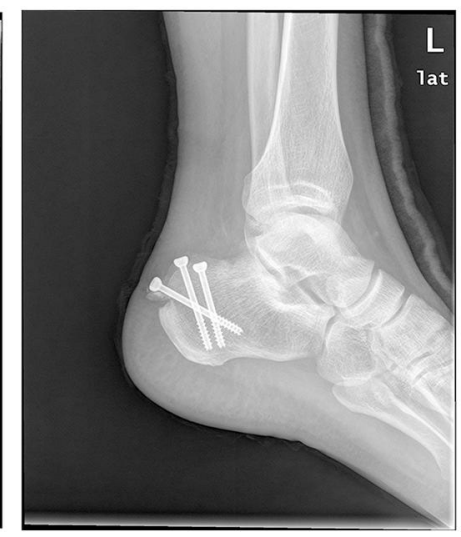

C

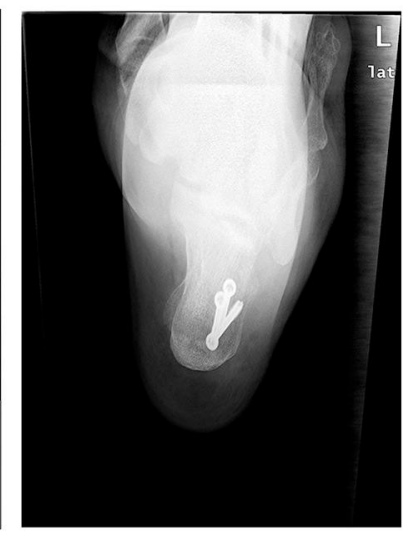

Figure I (A) Lateral X-ray of calcaneus, obvious displacement of calcaneal tuberosity. (B) Lateral X-ray of the calcaneus after the first operation, the fracture was fixed by three hollow lag screws. (C) Axial X-ray of the calcaneus after the first operation, and the screw can be fixed in position. 


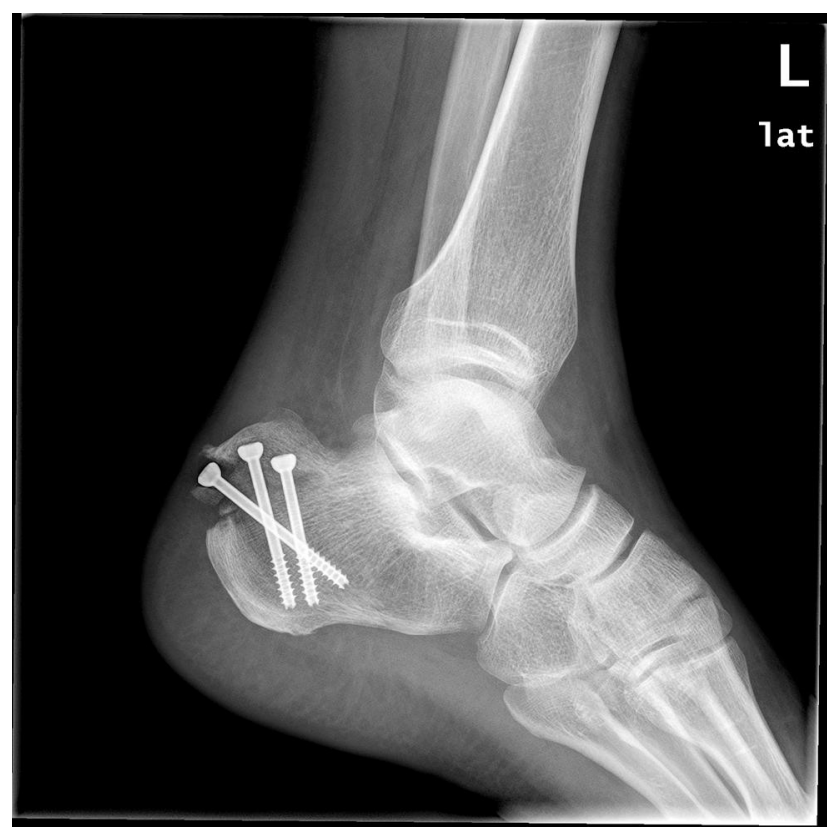

Figure 2 Lateral X-ray of the calcaneus, the avulsion fracture of the calcaneal tuberosity was displaced again, and the internal fixation failed.

A
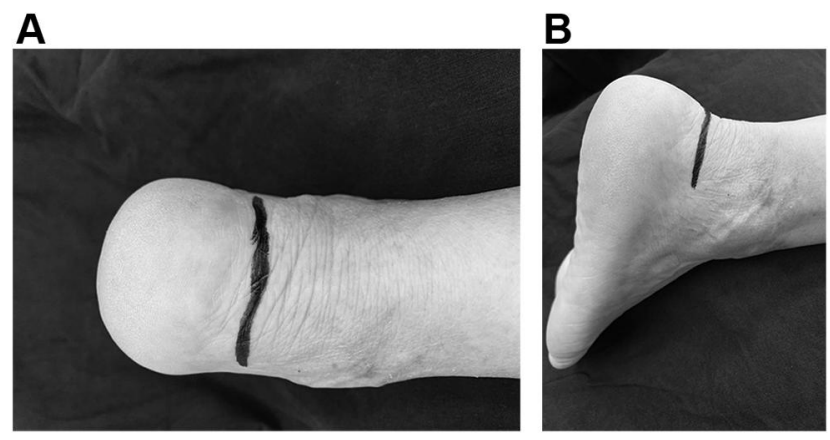

Figure 3 (A and $\mathbf{B}$ ) $5 \mathrm{~cm} \mathrm{U}$-shaped incision along the posterior ankle dermatoglyph at the level of the calcaneal tubercle.

\section{Results}

The patient underwent extensive physiotherapy immediately after surgery. The incision healed well and the patient was discharged about 1 week after the operation. He was regularly reviewed in the outpatient clinic and underwent imaging examinations (Figure 4). The patient started walking with the aid of crutches 1 month after surgery. Re-examination with computed tomography (CT) confirmed that the fracture reached the clinical healing standard and the patient started walking with full weight bearing 3 months after surgery. Twelve months after the operation, he was found to have full range of movement of his left ankle and no pain. The imaging examination showed that the fracture was well reduced and bone union was obtained. The case had an AOFAS score of 8012 months after surgery.

\section{Discussion}

The calcaneal tubercle is an important part of the calcaneus, which is of great significance in maintaining the arch of the foot and buffering weight. At the same time, it is the attachment point of the Achilles tendon. When we walk normally, the maximum load on the Achilles tendon is three times the body's weight. Therefore, the calcaneal tuberosity is prone to avulsion fractures when subjected to a certain external force. Studies have shown that the blood supply to the calcaneal tuberosity is poor, most of which are the terminal branches of small arteries and rarely form arterial networks. Moreover, these small arteries gradually decrease with age. ${ }^{12}$ At the same time, coupled with the compression of the displaced fractures on the skin, the soft tissues in this area are prone to necrosis. Early fracture reduction can relieve the pressure on the skin; restore local blood supply; reduce the incidence of complications, such as soft tissue necrosis; and restore the function of the calf triceps as soon as possible.

Open reduction is the first choice for avulsion fracture of the calcaneal tuberosity, and we should avoid repeated manual reduction. In reality, it is very difficult for us to reduce the displaced avulsion fracture of the calcaneal tuberosity in the closed state, and multiple reduction will cause repeated damage to local soft tissues and increase the probability of skin necrosis. Gardner et al ${ }^{10}$ reported 139 cases of posterior calcaneal tubercle fractures. Due to the traction of the Achilles tendon, the tongue-shaped fracture was displaced proximally, which increased the tension of the skin at the heel. Among them, 21\% of the cases had varying degrees of skin irritation on the back of the calcaneus. Ten patients had partial thickness flap injuries, and seven patients had complete full-thickness flap injuries. Two cases underwent free flap transplantation, and one case underwent amputation. Squires et $\mathrm{al}^{9}$ also believed that the effect of non-surgical treatment for avulsion fracture of the calcaneal tuberosity was unsatisfactory and open reduction and internal fixation was recommended. There are many studies on the choice of internal fixation for surgery. Squires et $\mathrm{al}^{9}$ and $\mathrm{Levi}^{13}$ recommended the use of steel wire and tension bands for fixation. Yoshida et $\mathrm{al}^{14}$ reported that lag screws were used to fix calcaneal tuberosity avulsion fractures with good results. Agni et al ${ }^{15}$ applied the hook plate to the avulsion fracture of the calcaneal tuberosity and found that it can also be firmly fixed. Lui ${ }^{6}$ believed that the stop point of the Achilles tendon was located at the posterior tubercle of the 

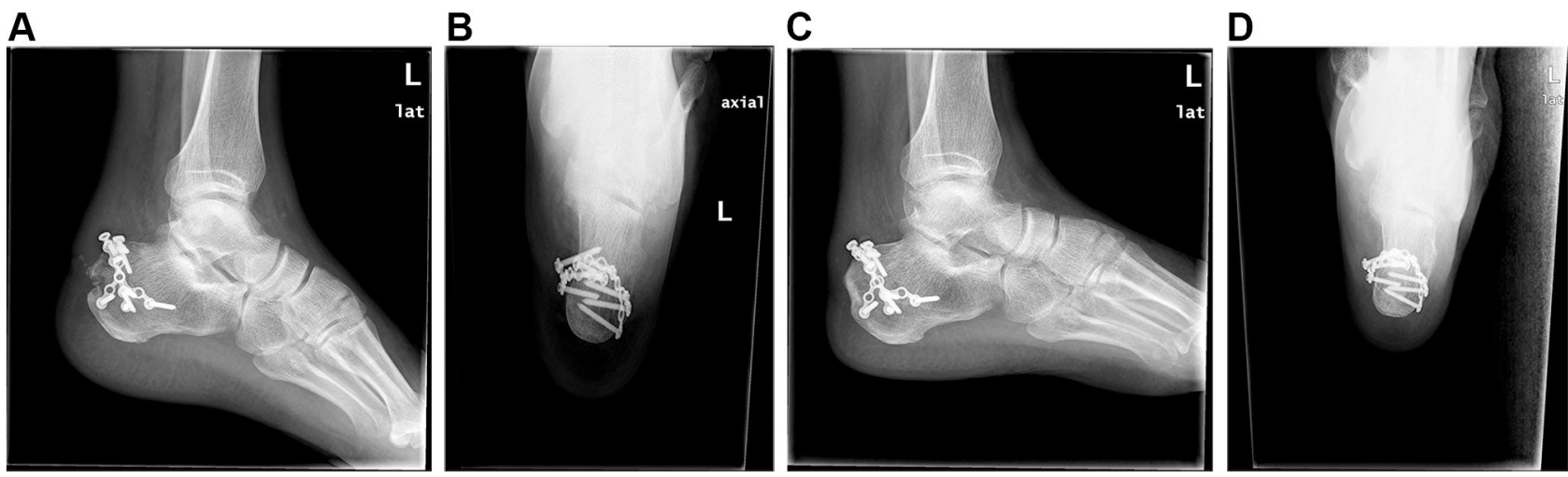

Figure 4 (A) The postoperative X-ray lateral radiograph, the displaced fracture was reset again after revision, and the mini-locking plate was fixed in a 180 -degree ring. (B) Postoperative axial X-ray of the case, the fractured piece was reliably fixed by the micro locking plate. (C) The X-ray lateral radiograph one year after the operation, the displaced fracture has been reset and achieved bony union, and the mini-locking plate was fixed in a 180-degree ring without loosening and displacement. (D) Axial X-ray one year after surgery, the fracture has reached bone union.

calcaneus. In order to restore the normal biomechanical function of the Achilles tendon, surgical treatment was recommended. However, screw fixation should be avoided because the mechanical strength of the screw was inadequate and the tail easily irritated the Achilles tendon. The above authors' studies are all case reports, and there is still a lack of research reports on large samples. So far, there is no widely accepted surgical method to treat avulsion fractures of the calcaneal tuberosity.

The 180-degree annular internal fixation with miniplate presented by us provides a new choice for surgeons in the treatment of avulsion fractures of the calcaneal tuberosity. This surgical technique has three main advantages:

1. The U-shaped incision is less intrusive to the soft tissue. It runs along the skin stripes to minimize the impact of postoperative scars on activities.

2. The mini-locking steel plate is small in size and will not compress the skin of the heel.

3. The 180-degree annular internal fixation and screw cross-locking and anchoring further enhance the fixing strength, which is convenient for early functional exercise.

There are many types of surgical incisions for avulsion fractures of the calcaneal tuberosity, usually including posteromedial incision, posterolateral incision, posterior midline longitudinal incision and L-shaped incision. These incisions are often perpendicular to the skin's texture so that later scar formation will have an adverse effect on the mobility of the ankle joint. The U-shaped incision we use can completely cut along the skin's texture, extending $1.5-2.0 \mathrm{~cm}$ from the Achilles tendon to both sides, which can fully exposes the fracture and help to complete a series of operations such as reduction and internal fixation. The study of Eren et $a^{16}$ also confirmed our statement that this type of incision can significantly reduce the incidence of incision-related complications. The steel plate we use is a micro-locking steel plate (Weigao, Shandong, China), which is small in size. Even if it is tightly fixed under the skin, it will not cause obvious compression on the skin. At the same time, there is a space between the stop point of the Achilles tendon and the posterior tubercle of the calcaneus to allow the steel plate to be placed after shaping, and it will not cause compression on the skin of the heel. If the fixing strength of one steel plate is not enough because the fracture piece is large and the displacement is obvious, another microplate can be added behind the Achilles tendon stop point, and there is no need to worry about the incision caused by its entrapment of the skin.

The failure of internal fixation is clearly related to bone quality. ${ }^{7,17}$ Athavale et al ${ }^{18}$ proved that the posterior tuberosity of the calcaneus is one of the sites with good bone quality and mechanical properties. Till ${ }^{19}$ also suggested that the screw should be fixed to the part of the posterior tuberosity of the calcaneus with good bone quality. Therefore, we imagine that the locking plate can be shaped and fixed to the cortex of the posterior tubercle during the operation to obtain a firm fixation of the fracture. The mechanical advantages of locking plates have been accepted by surgeons. ${ }^{19,20}$ On the basis of locking fixation, we rely on the good plasticity of the mini-locking plate to 
pre-bend the plate according to the specific shape of the fracture during the surgery. At the same time, we insert the screws in a 180-degree circle to further strengthen the firmness and reliability of the fixation. During the operation, we put the steel plate as close as possible to the cortical bone of the calcaneal tuberosity, and pre-bend the two ends of the steel plate to form a "U"-like structure that wraps the fractured piece of the posterior tuberosity of the calcaneus. When inserting the screws, we should pay attention to the arrangement of the cross anchoring. The screws should be long enough to lock and fix the double cortex. In this way, it can provide more powerful mechanical strength and achieve the purpose of early postoperative functional exercise, especially for elderly patients with osteoporosis.

Beavis et $\mathrm{al}^{21}$ divided the avulsion fractures of the calcaneal tuberosity into three categories: type $\mathrm{I}$ is a "sleeve-shaped" fracture, a small avulsion of cortical bone at the calcaneal tuberosity; type II is a "beak" fractures, an oblique fracture line running posterior from just behind Bohler's angle, but the Achilles tendon is still connected to the fracture; and type III is an avulsion fracture of the Achilles tendon from the middle third of the posterior tuberosity. Lee et $\mathrm{al}^{22}$ formulated corresponding treatment plans according to different fracture types. For type I and type II fractures, fixation with plate and screw is recommended, especially for type II fractures, which tend to have large fracture pieces and easily compress the skin. In reality, emergency reduction and fixation are often required for this type of fracture. For type III fractures, it is recommended that they be repaired with anchors. The surgical techniques we present are suitable for type I and type II fractures, especially for type II fractures. Because this type of fracture has large displacement and high fixation strength requirements, it is very suitable for the application of 180-degree circular locking and fixation technology.

\section{Strengths and Limitations}

For the first time, a new technique of 180-degree annular internal fixation was applied to avulsion fractures of the calcaneal tuberosity to observe its effects. However, our research is not fully free from limitations. First of all, our research was only a preliminary technical report. The sample size is small, and research with a large sample is needed. Moreover, the research on the biomechanical strength of the 180-degree circular fixation is temporarily blank, and we need to further strengthen the basic mechanical research in this area to verify the mechanical advantages of this technology.

\section{Conclusions}

In the treatment of avulsion fractures of the calcaneal tuberosity, there is currently no uniform standard surgical procedure. We presented a new technique of 180-degree annular internal fixation and achieved successful result. We hope that the application and promotion of this technology can reduce the failure rate of internal fixation of avulsion fractures of the calcaneal tuberosity and achieve the purpose of early functional exercise, especially for old patients with osteoporosis.

\section{Disclosure}

The authors report no conflicts of interest for this work.

\section{References}

1. Greenhagen RM, Highlander PD, Burns PR. Double row anchor fixation: a novel technique for a diabetic calanceal in-sufficiency avulsion fracture. J Foot Ankle Surg. 2011;51(1):123-127. doi:10. 1053/j.jfas.2011.09.006

2. Harb Z, Dachepalli S, Mani G. An alternative method of fixation of calcaneal tuberosity fractures using the Tightrope $\left({ }^{\circledR}\right)$ technique. J Foot Ankle Surg. 2013;52(6):762-765. doi:10.1053/j.jfas.2013.08. 005

3. Nagura I, Fujioka H, Kurosaka M, et al. Modified tension band wiring fixation for avulsion fractures of the calcaneus in osteoporotic bone: a review of three patient. J Foot Ankle Surg. 2012;51 (3):330-333. doi:10.1053/j.jfas.2011.10.049

4. Miyamoto W, Takao M, Matsui K, et al. Fixation for avulsion fracture of the calcaneal tuberosity using a side-locking loop suture technique and anti-slip knot. $J$ Foot Ankle Surg. 2015;36 (5):603-607.

5. Stapleton JJ, Kolodenker G, Zgonis T. Internal and external fixation approaches to the surgical management of calcaneal fractures. Clin Podiatr Med Surg. 2010;27(3):38 1-92. doi:10.1016/j.cpm.2010.03. 003

6. Lui TH. Fixation of tendo Achilles avulsion fracture. Foot Ankle Surg. 2009;15:58-61. doi:10.1016/j.fas.2008.06.004

7. Stoffel K, Booth G, Rohrl SM, et al. A comparison of conventional versus locking plates in intraarticular calcaneus fractures: a biomechanical study in human cadavers. Clin Biomech. 2007;22:100-105. doi:10.1016/j.clinbiomech.2006.07.008

8. Robb C, Davies MB. A new technique for fixation of calcaneal tuberosity avulsion fractures. $J$ Foot Ankle Surg. 2003;9 (4):221-224. doi:10.1016/S1268-7731(03)00089-4

9. Squires B, Allen PE, Livingstone J, Atkins RM. Fractures of the tuberosity of the calcaneus. J Bone Jt Surg Br. 2001;83(1):55-61. doi:10.1302/0301-620X.83B1.0830055

10. Gardner MJ, Nork SE, Barei DP, et al. Secondary soft tissue compromise in tongue-type calcaneus fractures. J Orthop Trauma. 2008;22(7):439-445. doi:10.1097/BOT.0b013e31817ace7e

11. Hess M, Booth B, Laughlin RT. Calcaneal avulsion fractures:complications from delayed treatment. Am J Emerg Med. 2008;26f21:254. el-4.

12. Chappard C, Brunet-Imbault B, Lemineur G, et al. Anisotropy changes in post-menopausal osteoporosis:characterization by a new index applied to trabecular bone radiographic images. Osteoporos Int. 2005;16(10):1193-1202. doi:10.1007/s00198-004-1829-5 
13. Levi N, Garde L, Kofoed H. Avulsion fracture of the calcaneus: report of a case using a new tension band technique. J Orthop Trauma. 1997;11 (1):61-62. doi:10.1097/00005131-199701000-00019

14. Yoshida K, Kasama K, Akahane T. Avulsion fracture of the calcaneus treated with a soft anchor bridge and lag screw technique: a report of two cases. J Foot Ankle Surg. 2016;55(2):310-313. doi:10.1053/j. jfas.2014.09.038

15. Agni N, Fearon P. Calcaneal tuberosity fixation using a locking compression hook plate. J Foot Ankle Surg. 2016;55(4):891-893. doi:10.1053/j.jfas.2016.03.012

16. Eren A, Cift H, Ozkan K, et al. Transverse incision for calcaneal tuberosity avulsion fractures. J Foot Ankle Surg. 2012;51(1):13 3-134. doi:10.1053/j.jfas.2011.10.015

17. Redfern DJ, Oliveira ML, Campbell JT, Belkoff SM. A biomechanical comparison of locking and nonlocking plates for the fixation of calcaneal fractures. Foot Ankle Int. 2006;27:196-201. doi: $10.1177 / 107110070602700308$
18. Athavale SA, Joshi SD, Joshi SS. Internal architecture of calcaneus: correlations with mechanics and pathoanatomy of calcaneal fractures. Surg Radiol Anat. 2010;32:115-122. doi:10.1007/s00276-009-0563-2

19. Illert T, Rammelt S, Drewes T, Grass R, Zwipp H. Stability of locking and non-locking plates in an osteoporotic calcaneal fracture model. Foot Ankle Int. 2011;32(3):307-313. doi:10.3113/FAI.2011. 0307

20. Blake MH, Owen JR, Sanford TS, Wayne JS, Adelaar RS. Biomechanical evaluation of a locking and nonlocking reconstruction plate in an osteoporotic calcaneal fracture model. Foot Ankle Int. 2011;32(4):432-436. doi:10.3113/FAI.2011.0432

21. Beavis RC, Rourke K, Court-Brown C. Avulsion fracture of the calcaneal tuberosity: a case report and literature review. Foot Ankle Int. 2008;29(8):863-866. doi:10.3113/FAI.2008.0000

22. Lee S-M, Huh S-W, Chung J-W, et al. Avulsion fracture of the calcaneal tuberosity: classification and its characteristics. Clin Orthop Surg. 2012;4(2):134-138. doi:10.4055/cios.2012.4.2.134
Clinical Interventions in Aging

\section{Publish your work in this journal}

Clinical Interventions in Aging is an international, peer-reviewed journal focusing on evidence-based reports on the value or lack thereof of treatments intended to prevent or delay the onset of maladaptive correlates of aging in human beings. This journal is indexed on PubMed Central, MedLine, CAS, Scopus and the Elsevier

\section{Dovepress}

Bibliographic databases. The manuscript management system is completely online and includes a very quick and fair peer-review system, which is all easy to use. Visit http://www.dovepress.com/ testimonials.php to read real quotes from published authors. 